\title{
Endoscope-assisted (with robotic guidance and using a hybrid technique) interhemispheric transcallosal hemispherotomy: a comparative study with open hemispherotomy to evaluate efficacy, complications, and outcome
}

\author{
P. Sarat Chandra, MCh, ${ }^{1,3}$ Heri Subianto, MD, ${ }^{1,3}$ Jitin Bajaj, $M C h,{ }^{1,3}$ Shabari Girishan, $M C h,{ }^{1,3}$ \\ Ramesh Doddamani, MCh, ${ }^{1}$ Bhargavi Ramanujam, DM, ${ }^{2,3}$ Mahendra Singh Chouhan, $\mathrm{MCh},{ }^{4}$ \\ Ajay Garg, MD, ${ }^{5}$ Madhavi Tripathi, MD, DNB,${ }^{6}$ Chandrasekhar S. Bal, MD, ${ }^{6}$ Chitra Sarkar, DM, ${ }^{7}$ \\ Rekha Dwivedi, PhD, ${ }^{2}$ Savita Sapra, DM, ${ }^{8}$ and Manjari Tripathi, DM ${ }^{2,3}$ \\ Departments of ${ }^{1}$ Neurosurgery, ${ }^{2}$ Neurology, ${ }^{5}$ Neuroradiology, ${ }^{6}$ Nuclear Medicine, ${ }^{7}$ Neuropathology, and ${ }^{8}$ Pediatric \\ Neuropsychology, All India Institute of Medical Sciences, New Delhi; ${ }^{3} \mathrm{COE}$, Epilepsy, National Brain Research Centre and All \\ India Institute of Medical Sciences, New Delhi; and 4Department of Neurosurgery, Shalby Multi-Speciality Hospital, Ahmedabad, \\ India
}

OBJECTIVE Endoscope-assisted hemispherotomy $(\mathrm{EH})$ has emerged as a good alternative option for hemispheric pathologies with drug-resistant epilepsy.

METHODS This was a prospective observational study. Parameters measured included primary outcome measures (frequency, severity of seizures) and secondary outcomes (cognition, behavior, and quality of life). Blood loss, operating time, complications, and hospital stay were also taken into account. A comparison was made between the open hemispherotomy $(\mathrm{OH})$ and endoscopic techniques performed by the senior author.

RESULTS Of 59 cases (42 males), 27 underwent $\mathrm{OH}$ (8 periinsular, the rest vertical) and 32 received $\mathrm{EH}$. The mean age was $8.65 \pm 5.41$ years (EH: $8.6 \pm 5.3$ years; $\mathrm{OH}: 8.6 \pm 5.7$ years). Seizure frequency per day was $7 \pm 5.9(\mathrm{EH}: 7.3 \pm$ 4.6; $\mathrm{OH}: 15.0 \pm 6.2$ ). Duration of disease (years since first episode) was $3.92 \pm 1.24$ years (EH: $5.2 \pm 4.3 ; \mathrm{OH}: 5.8 \pm 4.5$ years). Number of antiepileptic drugs per patient was $3.9 \pm 1.2(\mathrm{EH}: 4.2 \pm 1.2 ; \mathrm{OH}: 3.8 \pm 0.98)$. Values for the foregoing variables are expressed as the mean \pm SD. Pathologies included the following: postinfarct encephalomalacia in 19 (EH: 11); Rasmussen's syndrome in 14 (EH: 7); hemimegalencephaly in 12 (EH: 7); hemispheric cortical dysplasia in 7 (EH: 4); postencephalitis sequelae in 6 (EH: 2); and Sturge-Weber syndrome in 1 (EH: 1). The mean follow-up was 40.16 \pm 17.3 months. Thirty-nine of $49(79.6 \%)$ had favorable outcomes (International League Against Epilepsy class I and II): in $\mathrm{EH}$ the total was $19 / 23(82.6 \%)$ and in $\mathrm{OH}$ it was $20 / 26$ (76.9\%). There was no difference in the primary outcome between $\mathrm{EH}$ and $\mathrm{OH}(p=0.15)$. Significant improvement was seen in the behavioral/quality of life performance, but not in $\mathrm{IQ}$ scores in both $\mathrm{EH}$ and $\mathrm{OH}(\mathrm{p}<0.01$, no intergroup difference). Blood loss $(p=0.02)$ and hospital stay $(p=0.049)$ were less in $\mathrm{EH}$.

CONCLUSIONS EH was as effective as the open procedure in terms of primary and secondary outcomes. It also resulted in less blood loss and a shorter postoperative hospital stay.

https://thejns.org/doi/abs/10.3171/2018.8.PEDS18131

KEYWORDS endoscopic; epilepsy; hemimegalencephaly; hemispherotomy; minimally invasive

ABBREVIATIONS AED = antiepileptic drug; $\mathrm{CBCL}=$ Child Behavior Checklist; $\mathrm{EEG}=$ electroencephalography; $\mathrm{EH}=$ endoscope-assisted hemispherotomy; $\mathrm{HASS}=\mathrm{Hague}$ seizure severity; ILAE = International League Against Epilepsy; $\mathrm{OH}=$ open hemispherotomy; PedsQL = Pediatric Quality of Life; $\mathrm{QOL}=$ quality of life.

SUBMITTED February 28, 2018. ACCEPTED August 9, 2018.

INCLUDE WHEN CITING Published online November 9, 2018; DOI: 10.3171/2018.8.PEDS18131. 
$\mathrm{H}$ EMISPHEROTOMIES are usually performed through either a periinsular or a vertical parasagittal route, and many modifications have been described. ${ }^{10,12-15}$, 25,26,30,34,35,39 Recently, endoscope-assisted hemispherotomy (EH) has emerged as a viable minimally invasive option for performing hemispheric disconnection. , $2,4,6,8,36$ Following an initial technical description by the senior author (P.S.C.), ${ }^{4,8}$ the operation has now become the only procedure of choice at our institute. The objective of this paper was to consolidate the entire series of patients treated with $\mathrm{EH}$ and to compare the efficacy, outcomes, and complications in patients who underwent $\mathrm{EH}$ with those who received open hemispherotomy $(\mathrm{OH})$.

\section{Methods}

This is a prospective observational comparative study conducted between January 2007 and January 2017. The institutional review board granted approval, and patient consent was collected. The Strengthening the Reporting of Observational Studies in Epidemiology (STROBE) guidelines were implemented in the manuscript. Surgery was performed in all cases by the senior author at a single institution. The senior author has been performing hemispherotomies since 2001; hence, a significant period of learning was present prior to this study. ${ }^{5}$ Preoperative workup ${ }^{4,7,37}$ included an interictal electroencephalography (EEG), video EEG, MRI (1.5/3.0 T as per epilepsy protocol-Magnetom Espree, Siemens Medical Solutions), ictal SPECT, FDG-PET, and/or magnetoencephalography (Neuromag, TRIUX, Elekta), ${ }_{, 2}$ and a comprehensive epilepsy surgery meeting. Based on radiology results, pathologies were classified broadly into atrophic and nonatrophic. Criteria for inclusion in the atrophic group included the following: widening of the cortical sulci compared to the normal side, especially around the sylvian sulcus; dilatation of the ipsilateral lateral ventricle and temporal horn; and presence of a large porencephalic cyst due to postinfarct sequelae. Pathologies such as postinfarct porencephaly and some cases of advanced Rasmussen's disease were included in the atrophic group, whereas hemimegalencephaly, hemispheric cortical dysplasia, Rasmussen's disease without cortical atrophy, and Sturge-Weber syndrome were included in the nonatrophic group.

The surgical technique has been described previously. ${ }^{2,4}$ Briefly, the procedure was performed as follows (Fig. 1). A transverse 5-cm skin incision was made anterior to the coronal suture and a bone flap of $4 \times 3 \mathrm{~cm}$ was raised in the parasagittal location on one side of the midline. Neuronavigation (with Gd-enhanced MRI) was used in all cases to avoid bridging veins. The dura mater was opened in a $\mathrm{C}$-shaped manner with the base over the sinus. The ipsilateral hemisphere was retracted, and a rigid $0^{\circ}, 310-\mathrm{mm}-$ long, 10-mm-thick endoscope (Karl Storz, GmbH \& Co. $\mathrm{KG}$ ) was used for visualization. The endoscope was held with a robotic device (ROSA, Medtech), which doubled as both a holder and a neuronavigation device (Fig. 2). The endoscope with its holder was arranged in such a manner that the surgeon could be seated comfortably with optimal ergonomics.

This approach is especially important for this proce- dure, because it requires very fine dissection during some parts of the surgery. Our hybrid technique consists of using a 10 -mm-thick, 310-mm-long endoscope. This instrument is placed slightly inside the cranial opening (Fig. 3). Increasing the magnification allows the surgeon to visualize areas closer to the target site. This ability greatly reduces the chance of fogging and maintains excellent visual clarity. The technique is not comparable to an exoscope system, because the device is actually inside the cranial opening. Similarly, it is not comparable to a traditional endoscope-assisted surgery because the device is still away from the target, and the working instruments are quite distal to the telescopic lens. Using this scope there is some movement, which is performed depending on the depth of the surgery, but not as much as in a traditional endoscope-assisted surgery. The change in magnification serves to zoom in to the target site as well as zoom out to get a bird's-eye view.

The robotic device also provided haptic feedback, which is defined as the "use of the sense of touch in a user interface design to provide information to an end user." 19,31 When referring to mobile phones and similar devices, this term generally means the use of vibrations from the device's vibration alarm to denote that a touchscreen button has been pressed. For example, the phone would vibrate slightly in response to the user's activation of an on-screen control, making up for the lack of a normal tactile response that the user would experience when pressing a physical button. The resistive force that some "force feedback" joysticks and video game steering wheels provide is another form of haptic feedback. ${ }^{32}$

In addition, following the release of the locking system for the robot, there was no sudden increase in weight on the surgeon's hands, which would be expected to happen with a traditional endoscopic holding system. Thus, the robot was able to judge the feedback from the hand and accordingly aid movement in the intended direction. Also, the device enabled the surgeon to use it with just one hand while holding the instruments with the other hand. The robot thus allowed the scope to be maneuvered in any direction with minimal force due to the active magnetic release present within the system. This benefit enhanced the safety of surgery by preventing any damage to vital structures due to sudden, unpredictable movements that could happen if the entire weight of the system were to rest on the surgeon's hands. For lack of a better word, we used "haptic" to describe this process. The trajectory was also predefined on the ROSA robot, allowing optimal access.

An interhemispheric approach was performed, and surgery was conducted in 3 basic steps: 1) complete corpus callosotomy; 2) anterior and middle disconnection; and 3) posterior disconnection (Fig. 4). These steps are outlined in Video 1 and in Fig. 1 (see figure legend for details).

VIDEO 1. Clip showing the technique of EH. Copyright P. Sarat Chandra. Published with permission. Click here to view.

Following surgery, intraoperative MRI was performed to confirm complete disconnection (15/32), and a postoperative MRI sequence was performed for all patients at 3-6 months (Figs. 5-7) and again at 1 year. Special instruments included variable-impedance, low-temperature, nonstick, long bipolar forceps (radiofrequency [e.g., Sutter 

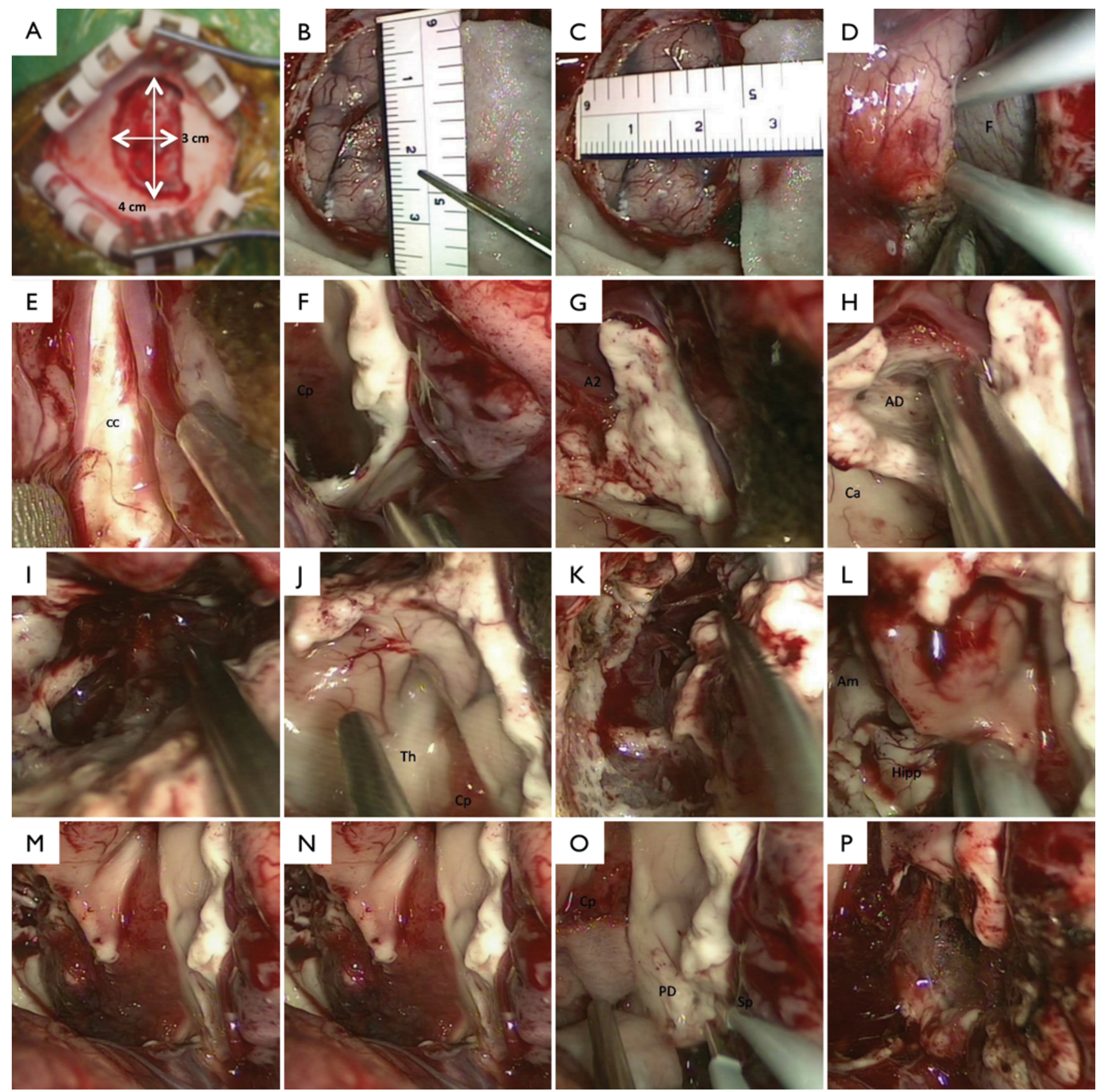

FIG. 1. Photographs showing the operative steps of EH. A: The size of skin incision, which was approximately $5 \mathrm{~cm}$ along the coronal plane. All photographs except panel A were taken from the endoscope (the usual ring is not seen because the view is magnified). The skin incision was placed anterior to the coronal suture, and the exact site was decided by neuronavigation to avoid bridging veins. The bone flap was $4 \times 3 \mathrm{~cm}$ (sagittal $\times$ coronal) and is raised on one side of the midline. B and C: The dura was opened in a C-shaped manner with the base toward the midline. D: The brain may look full initially. However, gentle retraction of the hemisphere followed by gradual and patient release of CSF will lead to the brain becoming lax, and the interhemispheric fissure opens up like a book. It is also important to understand that the procedure follows the principles of keyhole surgery, in which there is more retraction in the deeper part than on the surface (expanding cone). $\mathrm{F}=$ falx. $\mathrm{E}$ : The corpus callosum (cc) is gently exposed from the genu toward the posterior part between both of the anterior cerebral arteries (ACAs). F: The corpus callosum is then divided; first the posterior part, followed by the splenium (which is curving inferiorly and hence can be divided once the posterior part is divided), and then finally followed by genu. It is important to split the corpus callosum so that the ipsilateral ventricle is entered ( $\mathrm{Cp}=$ choroid plexus). $\mathbf{G}$ : The junction of the genu and the ACA is then exposed. $\mathrm{H}$ : The anterior disconnection (AD) is then started at the junction of the ACA and genu anterior to the ventricle. This disconnection is now extended laterally along the coronal plane, anterior to the head of the caudate nucleus (Ca). I: The anterior disconnection is then deepened inferiorly until the posterior part of the anterior cranial fossa is reached. It is extended laterally until the lateral part of the head of caudate nucleus is reached. FIG. 1. (continued) $\rightarrow$ 
FIG. 1. J: The middle disconnection is now started at the lateralmost part of the anterior disconnection and passes lateral to the putamen and thalamus (Th). K: Progress of the middle disconnection, which is deepened and exposes the sphenoid ridge and the middle cerebral artery anteriorly and opens into the temporal horn posteriorly. L: Both the amygdala (Am) and the hippocampus (Hipp) can be seen in this figure. M: The choroid plexus along the choroid fissure as it passes to the temporal horn (as it is seen after the middle disconnection) is completed lateral to the thalamus. $\mathbf{N}$ : Following the middle disconnection, the next important step is to aspirate the ventral amygdala and the anterior part of hippocampus so that it is disconnected from the dorsal amygdala, effectively disconnecting the anterior medial part of the temporal lobe from the diencephalic structures. 0: The last part of the hemispheric disconnection is the posterior disconnection (PD), which passes along the line connecting the splenium (Sp) laterally and the choroid plexus medially. P: This technique disconnects the temporal efferents consisting of the tail of the hippocampus and the fornix. Figure is available in color online only.

Medizintechnik] or molecular resonance based-Vesalius; Medilife Technologies); long suction cannulas, preferably with tapering and detachable tips; and/or suction integrated bipolar forceps (Karl Storz, GmbH \& Co. KG). We found bayoneted irrigated bipolar forceps (Aquamantys; Medtronic) to be useful, especially to reach the deeper areas during the last part of the middle disconnection. Alternatively, long bipolar forceps also helped in performing the middle disconnection.

The middle disconnection was the most difficult aspect of the surgery for two reasons. First, this area had the largest depth of working length. Second, the presence of lenticulostriate vessels in this area just lateral to the lentiform nucleus causes bleeding. This issue may not be substantial, but it may be troublesome because the vessels are very fine and sometimes have difficulty obtaining hemostasis. At this stage, we generally find it convenient to use the variableimpedance bipolar forceps. Here, neuronavigation was also useful to prevent transgression of the midline diencephalic structures, because there was minimal or no shift in these structures during surgery. In nonatrophic cases, we found it convenient to aspirate out more brain parenchyma during disconnection to create space during surgery. Neuronavigation was very useful in these cases, especially when ventricular spaces were not formed. Following surgery, an intraventricular drain was placed for a period of 72 hours. If required, elective ventilation was performed overnight.

Primary outcome measures included the assessment of seizure frequency and severity as per the International League Against Epilepsy (ILAE) scale ${ }^{41}$ and the Hague seizure severity (HASS) ${ }^{3}$ scale, respectively. These outcomes were assessed in a blinded fashion. Secondary outcome variables were cognition, behavior, and quality of life (QOL): the Binet Kamat test/Vineland social maturity scale (BKT/VSMS) was administered to assess IQ/social quotient (IQ/SQ); Child Behavior Checklist (CBCL) to as-

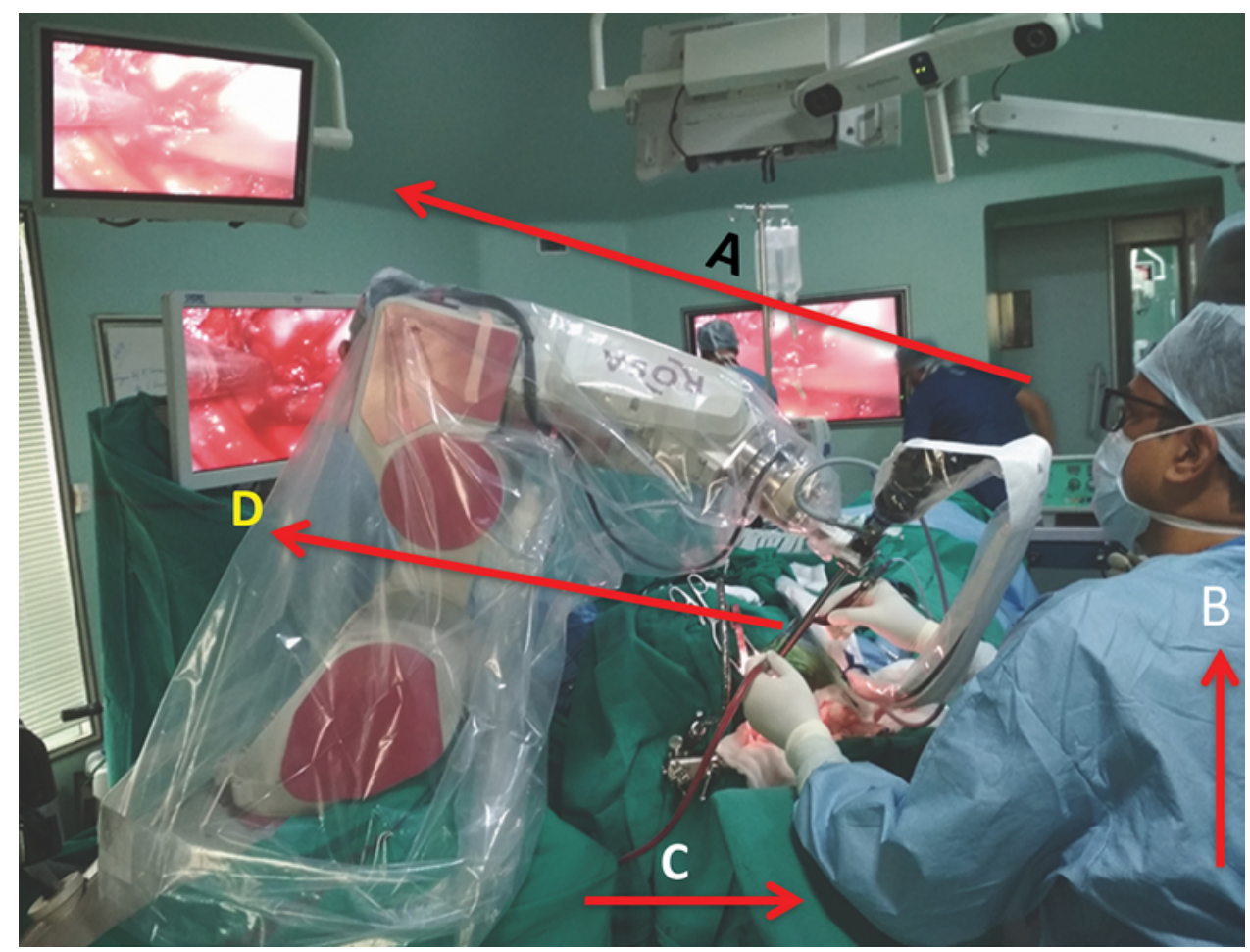

FIG. 2. The operating room setup of the endoscope with the robotic device. It is important for the surgeon to be seated comfortably so that an optimal visual axis $(A)$ is maintained along with proper back support (B). It is also important to have proper elbow support (C). The endoscope (D) uses a hybrid technique as described in the text. Proper ergonomics are important to enhance accuracy of the surgery and prevent surgeon exhaustion. Figure is available in color online only. 


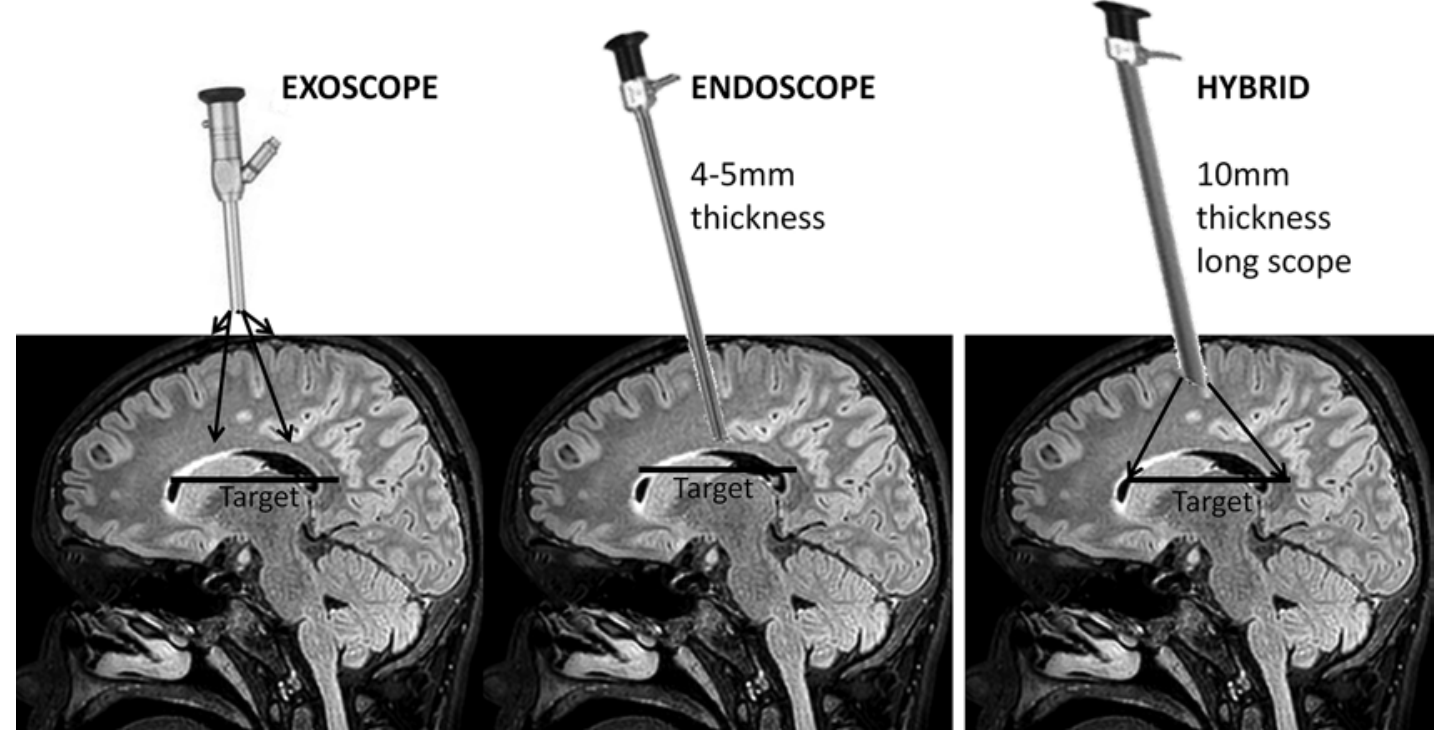

FIG. 3. The fundamental differences between an exoscope, endoscope-assisted surgery, and the hybrid technique proposed by us. While using an exoscope, the telescope is outside the cranial opening, and this leads to dissipation of the light, which may result in inadequate visualization in the depth. In endoscope-assisted surgery, the endoscope $(4-5 \mathrm{~mm})$ has to be kept close to the target. This may lead to frequent opacification of the lens because of blood. This system nevertheless provides high magnification, but leads to loss of a bird's-eye view of the target. Endoscope-assisted surgery may be suitable for pituitary tumors, where the target is approximately $10-15 \mathrm{~mm}$ in size. In hemispherotomy, the target is several centimeters in size (from genu to splenium), and thus requires frequent changes of view from a bird's-eye to a close-up view. To circumvent these problems, we have introduced the concept of a hybrid technique, in which a 10-mm-thick, 310-mm-long scope is used. Although the scope is inside the cranial opening, it is still away from the target. The working instruments such as the suction and bipolar forceps would be distal to the telescope. With the increased thickness of the scope, the visualization becomes better, and the target and the surrounding areas may be visualized easily by changing the magnification. The main advantages of this system are as follows: 1) it allows very good visualization of the target without any diffusion of light extracranially; 2 ) it does not impede the working of the instruments because the telescope is proximal to them, thus preventing frequent opacification of the lens by blood; and 3) by altering the zoom, the surgeon can rapidly change from magnified view to a bird's-eye view. Thus, the hybrid system has the advantages of the endoscope and exoscope while avoiding the disadvantages of both.

sess behavioral problems; and Pediatric Quality of Life (PedsQL) scale to assess QOL. Seizure outcome (ILAE class I and II) was grouped as favorable, and ILAE class III and higher was considered an unfavorable outcome. Follow-up in the form of primary outcome measures (seizure log, adverse events) was performed at monthly intervals for 12 months and then every 3 months up to 60 months (a structured questionnaire performed via telephone call). Secondary outcomes were analyzed at 6 months and 1 year. In addition, a subjective 10-point questionnaire was completed by the patient's parents at follow-up for patients who underwent $\mathrm{EH}$.

Data were analyzed using IBM SPSS version 20.0 (IBM Corp.); values are expressed as the mean $\pm \mathrm{SD}$. The chi-square/Fisher's exact test was used to compare the $\mathrm{OH}$ and $\mathrm{EH}$ groups with respect to the baseline variable and categorical variables. Log-rank test was used to compare the primary outcomes between the groups. Independent t-test or the generalized estimating equation test was used to compare the secondary outcome variables between the 2 groups and within the groups at different follow-up time points. A p value $<0.05$ was considered significant, and the confidence interval was calculated if required. In addition, operating time (from time of skin incision until the last suture was applied), blood loss (calculated by subtracting the irrigation saline taken from the drain fluid and weighing the gauges before and after the surgery), and hospital stay were also compared. All adverse events were noted and recorded. Worsening of hemiparesis or development of hemiparesis/hemiplegia was considered an anticipated consequence and not a complication, as proposed by Rydenhag and Silander. ${ }^{33}$ Following surgery, all antiepileptic drugs (AEDs) were continued. Tapering was considered only after 1 year. For congenital pathologies such as hemimegalencephaly, AEDs were continued (albeit in reduced dosage).

\section{Results}

Of 59 cases (42 males), 27 underwent $\mathrm{OH}$, and 32 underwent $\mathrm{EH}$. The $\mathrm{OH}$ procedures were performed until March 2013, and included microscopic periinsular (8) or vertical interhemispheric transcallosal (19) approaches. The EHs were performed from April 2013 onward.

\section{Demographic Data}

The patients' mean age was $8.65 \pm 5.41$ years (range $0.4-25$ years; EH: $8.6 \pm 5.3$ years; $\mathrm{OH}: 8.6 \pm 5.7$ years). Seizure frequency per day was $7 \pm 5.9$ (EH: $7.3 \pm 4.6[1$ status epilepticus]; $\mathrm{OH}$ : $15.0 \pm 6.2$ ). Age at onset of sei- 


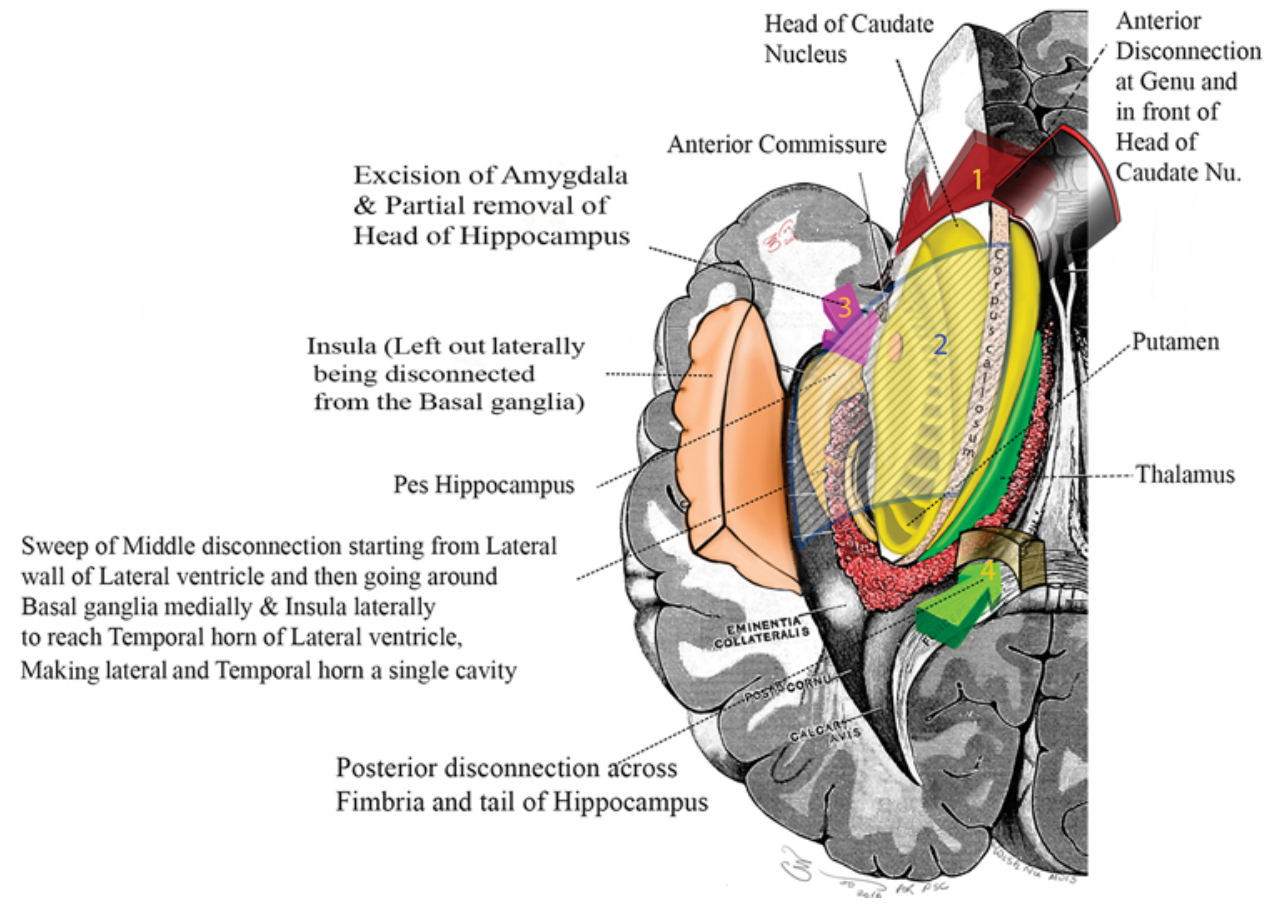

FIG. 4. Schematic diagram with embedded labels showing the anterior disconnection (1), middle disconnection (2), excision of the ventral amygdala (3), and the posterior disconnection (4). The diagram is self-explanatory and follows the surgical steps as shown in Fig. 1. Nu. = nucleus. Copyright Mahendra Singh Chouhan. Published with permission. Figure is available in color online only.

zures was $33.3 \pm 36.9$ months (range $0.03-168$ months; EH: $35.4 \pm 39.08$ months; OH: $29.2 \pm 33.3$ months). The mean duration of disease at the time of surgery (years since the first episode) was $3.92 \pm 1.24$ (EH: $5.2 \pm 4.3 ; \mathrm{OH}$ : $5.8 \pm 4.5)$. The number of AEDs per patient was $3.9 \pm 1.2$ (EH: $4.2 \pm 1.2$; OH: $3.8 \pm 0.98$ ). There was no significant intergroup difference.

\section{Semiology of Seizures}

Hemiparesis/hemiplegia (with pincer grip weakness) was present in $95 \%(\mathrm{n}=56)$. The most common seizure syndromes were complex partial seizures (74\%), simple partial seizures $(46.5 \%)$, and secondary generalized tonic/ clonic seizures $(39.6 \%)$. One child presented with status epilepticus, and an emergency EH was performed.

Other semiologies included delayed cry at birth in $31 \%$ $(\mathrm{n}=18)$, drop attacks in $20 \%(\mathrm{n}=12)$, status epilepticus in $18.6 \%(\mathrm{n}=11)$, epilepsia partialis continua in $17 \%(\mathrm{n}=$ 10), myoclonic jerks in $6.8 \%(n=4)$, and febrile seizures in $6.8 \%(\mathrm{n}=4)$. All patients had more than 1 seizure type $($ mean $=3)$.

\section{Neuroimaging Investigations}

All patients underwent MRI, interictal EEG, and video EEG. In addition, PET $(\mathrm{n}=28)$, SPECT $(\mathrm{n}=34)$, and magnetoencephalography were performed $(n=25)$.

\section{Pathologies}

Pathologies included postinfarct encephalomalacia in 19 (EH: 11); Rasmussen's syndrome in 14 (EH: 7); hemi- megalencephaly in 12 (EH: 7); hemispheric cortical dysplasia in 7 (EH: 4); postencephalitis sequelae in 6 (EH: 2); and Sturge-Weber syndrome in 1 (EH: 1). Among these pathologies, $54.23 \%(\mathrm{n}=32$; EH in 14) were categorized as atrophic pathologies, and the rest were nonatrophic $(\mathrm{n}=27$, 45.76\%; EH in 18). The early part of EH consisted of mostly atrophic pathologies to allow an adequate learning curve. ${ }^{4}$

\section{Seizure Outcome}

Of the total of 59 patients who underwent hemispherotomy (OH: 27, EH: 32), 49 (excluding 2 who died-1 in the $\mathrm{OH}$ group in the perioperative period, and 1 in $\mathrm{EH}$ after 9 months due to lung infection) had a minimum follow-up period of 1 year (OH: 26, EH: 23). The mean follow-up of these 49 patients was $40.16 \pm 17.3$ months $(\mathrm{OH}: 54.81 \pm$ 6.26 months; EH: $23.61 \pm 8.25$ months). A total of 20/26 (77\%) had favorable outcomes (ILAE class $^{41}$ I and II; i.e., seizure free with or without auras) in the $\mathrm{OH}$ group, as did 19/23 (82.6\%) in the EH group (total number in both groups was 39/49 [79.6\%]). There was no significant difference between both groups, and the p value was 0.15 (not significant). Figure 8 shows the Kaplan-Meier survival curves in relation to the seizure events. The ILAE class III outcome was seen in 5 of the remaining patients $(8.5 \%)$, class IV in 4 patients (6.8\%), and class V in 1 patient (1.7\%).

The preoperative HASS was $40.32 \pm 3.95$, and the postoperative HASS at 1 year was $17.6 \pm 4.7$. This difference was significant $(\mathrm{p}<0.001)$, but there was no difference between the 2 groups (EH and $\mathrm{OH})$.

The secondary outcome scores for IQ assessed at 6 months and 1 year showed some improvement (preopera- 

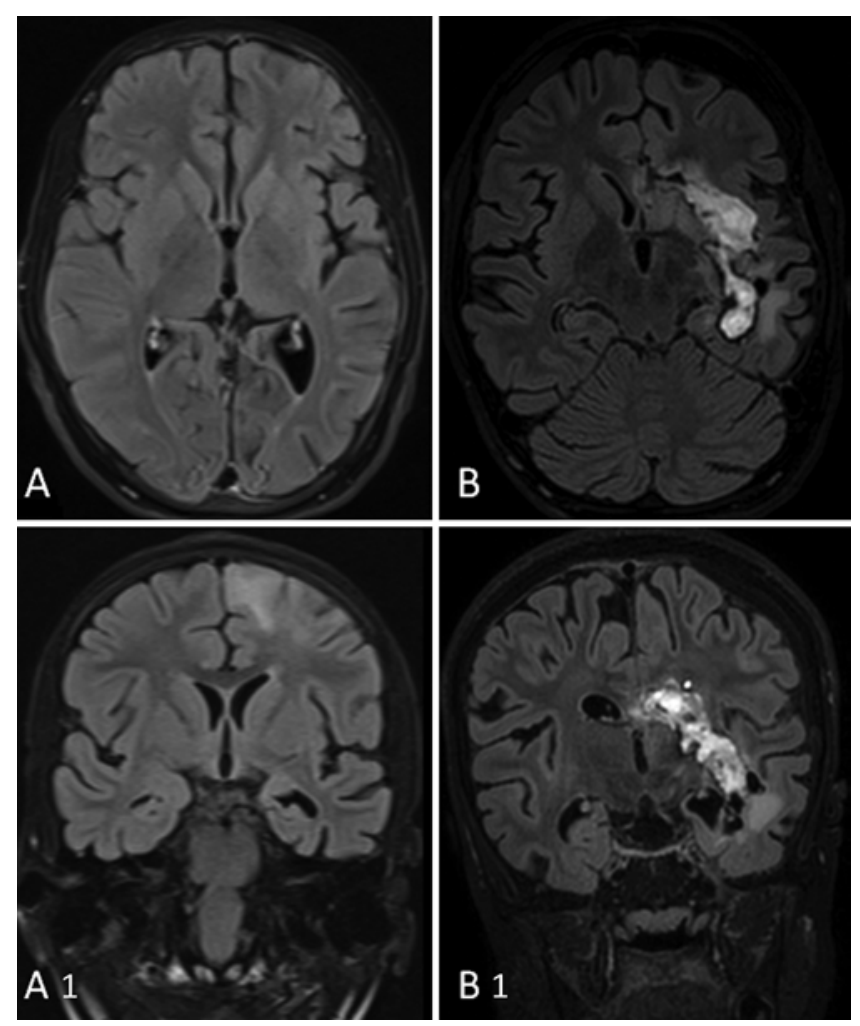

FIG. 5. A 9-year-old girl presenting with progressive increasing severity and frequency of focal seizure (1-3 times/day at the time of surgery) with delayed milestones and with right epilepsia partialis continua.

A: MRI sequences showed signal changes in the left hemisphere suggestive of Rasmussen's syndrome (example of a nonatrophic pathology). $\mathrm{B}$ : Following EH performed using neuronavigation she had an ILAE class IA outcome. This MRI sequence was performed 7 days after surgery and shows the line of disconnection in axial ( $A$ and $B$ ) and coronal (A1 and B1) sections.

tive: $54.57 \pm 24.62$; postoperative: $58.3 \pm 14.6$ ), but there was no significant difference. The PedsQL score improved significantly following surgery (total $\mathrm{T}$ score-preoperative: $43.6 \pm 7.1$; postoperative: $75.5 \pm 12.3[\mathrm{p}<0.01])$. The improvement was significant in all PedsQL domains (physical health summary: preoperative, $38.23 \pm 12.10$; postoperative, $77.0 \pm 14.30$; psychosocial health summary: preoperative, $48.37 \pm 8.54$; postoperative, $75.24 \pm 6.23$ ) There was no difference between the $\mathrm{EH}$ and $\mathrm{OH}$ groups for similar periods of time. The mean \pm SD total behavior problem score for the CBCL was $60.5 \pm 4.81$ at followup (the preoperative score was $79.0 \pm 6.31[\mathrm{p}>0.01$, not significant]; normal score $<60$ ). The secondary outcomes could not be recorded for 2 patients (both in the EH group, one presenting with status epilepticus and the other with severe morbidity—see Complications).

\section{Motor Outcome}

Of the 56 patients presenting with hemiparesis, power remained unchanged in 30 patients $(53.6 \%)$ and worsened in 26 (46.4\%; improved at follow-up in 22/26 [84.6\%]). Worsening motor function mostly occurred in older patients $(\mathrm{n}=12$, age $>13$ years). Two patients, who did not
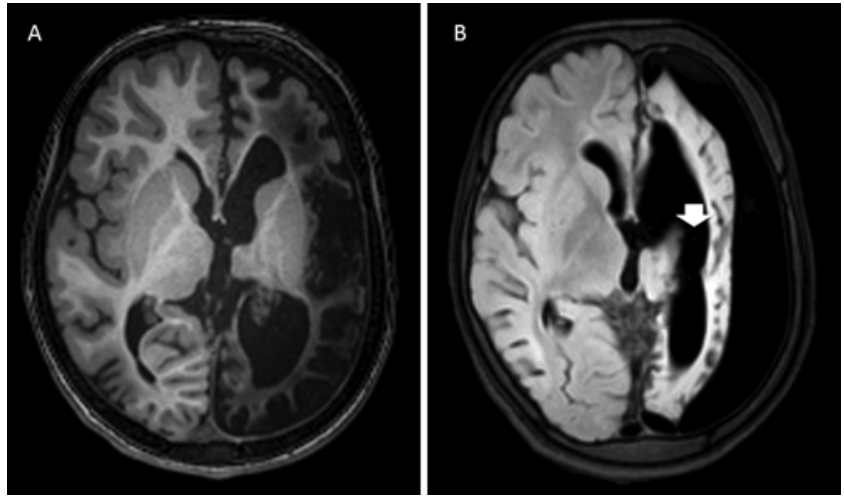

FIG. 6. A 3-year-old boy with Rasmussen's syndrome (A) who underwent EH. Repeat MRI sequence (B) performed at 1 year showed a deafferentation atrophy of the hemisphere. Arrow indicates the line of middle disconnection between the putamen and thalamus.
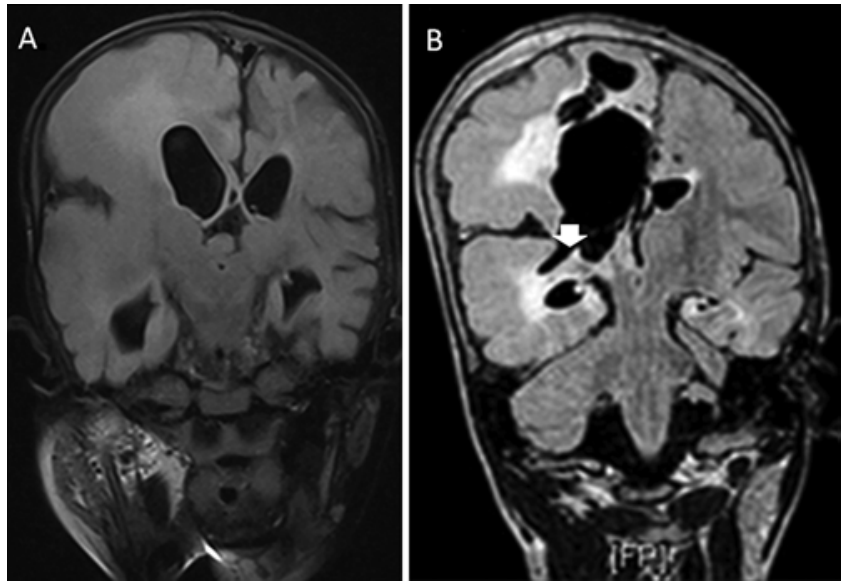

FIG. 7. A 9-month-old boy who had right hemimegalencephaly (A). Following $\mathrm{EH}$, the child had an ILAE class IA outcome at 1 year. However, the repeat MRI showed a persistent connection (arrow) at the level of the ventral amygdala (B). One may also notice a large cavity at the site of disconnection. It is likely that this bridge of tissue is nonfunctional. The child is being followed carefully. In the case of recurrence of seizures, an endoscopic approach may be undertaken to divide this bridge of tissue. This case illustrates the importance of adequate removal of the ventral amygdala and the anterior hippocampus to disconnect the anterior and mesial temporal structures completely from the midline structures.

have preoperative hemiparesis, were children with hemimegalencephaly. They developed some paucity of movement postoperatively, which improved in a few days. There was no difference between $\mathrm{OH}$ and $\mathrm{EH}$ in terms of motor outcome.

\section{Blood Loss}

The mean blood loss was $718.75 \pm 396.35 \mathrm{ml}$ in the $\mathrm{OH}$ group and $210.42 \pm 197.25 \mathrm{ml}$ in the $\mathrm{EH}$ group. This difference was significant $(\mathrm{p}=0.02,99 \% \mathrm{CI})$.

\section{Operating Time}

The mean duration of surgery was $351.25 \pm 44.781 \mathrm{~min}-$ 


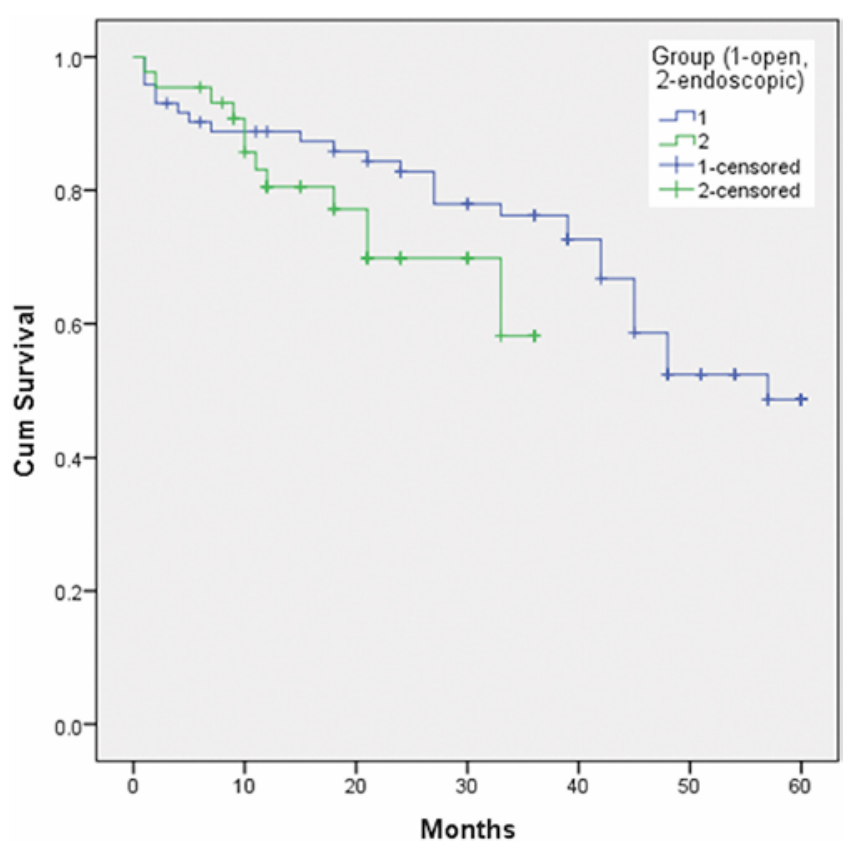

FIG. 8. Kaplan-Meier curves for the cumulative survival in relation to seizure events in the $\mathrm{OH}$ group (blue line) and EH group (green line). The $x$-axis is the number of months after the surgery, and the $y$-axis shows cumulative survival (i.e., patients without a single seizure after the surgery). Censored values (+) indicate the last known follow-up time. Differences between the 2 groups were not significant based on the log-rank test $(p=0.15)$. Using Fisher's exact test, the $p$ value was 0.451 at 12 months and 0.371 at 30 months; neither was significant. Figure is available in color online only.

utes in the $\mathrm{OH}$ group and $301.87 \pm 65.836$ minutes in the EH group. The difference was not significant $(p=0.06)$ but trended toward a decreased time in the EH group. The mean duration of surgery for the atrophic group was $252.85 \pm 59.83$ minutes and it was $308.18 \pm 64.12$ minutes for the nonatrophic group (significant at $\mathrm{p}=0.03,99 \% \mathrm{CI}$ ).

\section{Postoperative Hospital Stay}

The mean hospital stay in patients who underwent $\mathrm{OH}$ was $18.6 \pm 22.4$ days (range 7-122 days) and $14.6 \pm 6.7$ days (range $8-26$ days) in $\mathrm{EH}(\mathrm{p}=0.049,99 \% \mathrm{CI})$. This result does not include a patient in the EH group who had a very prolonged stay-for more than 2 months-due to a complication (see Complications).

\section{Questionnaire}

In the subjective questionnaire, the parents $(\mathrm{n}=19$ responses) overall expressed being "very satisfied" with the EH procedure and were quite happy with the cosmetic effects related to small size of scar, quick healing, less scalp swelling, and the scar becoming covered with hair growth. In addition, they also expressed a subjective feeling of "relief" on seeing the small size of the scalp incision following surgery.

\section{Complications}

There was 1 death (hemimegalencephaly in the $\mathrm{OH}$ group). This 3-year-old was being followed in an outpatient department and was awaiting admission when he presented to emergency services with status epilepticus, aspiration, hypotension, and altered sensorium. He was taken for surgery after stabilization. Following surgery, the child did not recover. An MRI study that was done after 1 week showed multiple infarcts.

There was 1 death in the EH group. This patient also came to the emergency department in status epilepticus with aspiration pneumonia. Following surgery, he remained tracheostomized. He was discharged in stable condition but succumbed after 9 months due to pneumonia.

Complications and/or adverse events occurred in 22 patients, with the most common being postoperative fever ( $\mathrm{n}$ $=18, \mathrm{EH}=10$ ). These patients had no evidence of infection, and the cause was considered to be aseptic meningitis due to the blood in the ventricles. In most cases, fever subsided within 1 week $(\mathrm{n}=10, \mathrm{EH}=6)$.

One patient in the $\mathrm{OH}$ group developed a large venous infarct following a periinsular hemispherotomy. He had to undergo decompressive craniotomy, and he gradually improved but had poor secondary outcome scores. Following this complication, we switched to an interhemispheric transcallosal approach for all cases, which was a precursor for $\mathrm{EH}$.

Additional complications included postoperative brain abscess ( $\mathrm{EH}=1$ [needed drainage and antibiotics, recovered completely]); meningitis $(\mathrm{OH}=1)$; and hyperammonemia $(\mathrm{OH}=1)$. Both of the latter 2 cases were treated appropriately and improved completely.

\section{Discussion}

\section{Primary Outcome Measures}

Hemispherotomy/hemispherectomy is the only option for patients with panhemispheric pathologies. ${ }^{18}$ This study has reviewed the results of both $\mathrm{OH}$ (mostly vertical interhemispheric) and EH (developed by the senior author) $)^{2,46}$ performed in 59 patients at different periods of time between 2007 and 2017. Of these cases, outcomes were analyzed for 49 patients (with at least 1 year of followup); $79.6 \%$ had a favorable outcome-ILAE class I or $\mathrm{II}^{41}$ (82.6\% for $\mathrm{EH}$ at $23.61 \pm 8.25$ months of follow-up, and $77 \%$ for $\mathrm{OH}$ at $54.81 \pm 6.26$ months of follow-up). There was no significant difference found between the groups. We started performing hemispherotomy in $2001 ; 5$ hence, an adequate time period was available to optimize skills before the start of this study. We switched from a periinsular procedure to a vertical interhemispheric procedure (a slight modification of the Delalande technique, ${ }^{12,13}$ which was parasagittal transcortical). The latter was a precursor to the endoscopic technique. This approach was used following a complication involving the development of a large venous infarct in 1 patient. Hence, this change in paradigm provided us an opportunity to compare both techniques.

Our results are similar in outcome to other studies, considering the fact that we have included ILAE classes I and II as favorable, although our follow-up period is not as long. Comparison with other studies is difficult, because the proportions of study subjects in each subtype of pathology are different. For instance, congenital pathologies 
such as hemimegalencephaly have worse outcomes than acquired pathologies such as postinfarct porencephaly, and the outcome of Rasmussen's falls in between.

\section{Secondary Outcome Measures}

There was no significant improvement in IQ following surgery for both $\mathrm{EH}$ and $\mathrm{OH}$. However, there was a significant improvement in behavioral scores (assessed using the CBCL). Other groups have also demonstrated similar results. ${ }^{11,16,20,24,29,38}$ It is unlikely that cognitive scores improve within a period of 1-2 years. Longer periods of follow-up have demonstrated improvement in cognitive performance scores. In this study, we measured secondary outcomes only at 1 year. As also demonstrated in other studies following hemispheric surgery, the parents or caregivers often note that the child becomes less violent and has significant quiescence in their temper tantrums. The association of behavioral disorders in children with hemiplegia and their improvement with hemispherectomy has been noticed previously. ${ }^{28,42}$ More contemporary studies ${ }^{9,20-22,27,40}$ using objective measures to assess behavioral disorders have demonstrated significant improvement in behavioral performance following hemispheric surgery.

Hemispheric surgery, whether open or endoscopic, is not a minor surgery. The risk of complications is known to increase with an increase in frequency and severity of preoperative seizures, with the presence of nonatrophic pathologies such as hemimegalencephaly, and in children younger than 18 months of age. The morbidity also increases with the presence of status epilepticus and comorbidities (like chest infection). It is thus advised that surgeons with less than optimal experience should not operate on these patients. The complications in our study compare with those described in other large studies. We believe that fever not associated with infection is a common phenomenon because it is also described in other large studies, and it should not be considered a complication like postoperative hemiparesis.

Although there were fewer complications in $\mathrm{EH}$, we think that the cohort is too small to comment on this result. There was 1 death in the $\mathrm{OH}$ group and none in the perioperative period in the $\mathrm{EH}$ group. Of significance is 1 case of a venous infarct (requiring decompressive craniotomy) that developed in the $\mathrm{OH}$ group (patient underwent periinsular hemispherotomy). This outcome is a significant complication in periinsular surgery, in which brain swelling leading to increased intracranial pressure may develop if adequate space is not created. This complication led us to change our surgical strategy to an open microscopic interhemispheric transcallosal approach. This technique was a precursor to the development of $\mathrm{EH}$, which is also advisable as a starting procedure for surgeons with less experience. The endoscopic route definitely has the advantage of going through an avascular corridor.

A significant advantage of EH was reduced blood loss compared to the $\mathrm{OH}$ group $(210.42 \pm 197.25 \mathrm{ml}$ vs 718.75 $\pm 396.35 \mathrm{ml}$, respectively). We think that this difference is because of smaller incisions and craniotomy, excellent visualization, and use of avascular routes for hemispheric disconnection. We believe that the occurrence of these factors is likely to be reduced further with an increase in expertise, and this is of significance considering the fact that this surgical procedure is primarily performed in children. For the same reasons, there was also a significant reduction in postoperative stay in the EH group (14.6 \pm 6.7 days compared with $18.6 \pm 22.4$ days in the $\mathrm{OH}$ group). We again think that this result was due to the small opening and minimal or no scalp swelling compared to large craniotomies performed in the $\mathrm{OH}$ group. This finding was reiterated by the positive responses we received from the subjective questionnaire.

\section{Surgical Technique}

The technique is similar to the principles described previously by Delalande et al., ${ }^{12}$ Dorfer et al., ${ }^{17}$ and Kawai et al., ${ }^{25}$ but it is different from their techniques in several ways, as follows:

1. In Kawai et al.'s technique, the corpus callosotomy is first performed at midline, and then the body of the lateral ventricle is opened. In our technique, we have suggested that the affected hemisphere should be retracted ipsilaterally and the corpus callosotomy performed in such a manner that the body of the lateral ventricle is directly opened.

2. In Kawai et al.'s technique, the anterior disconnection connects the anterior end of the choroidal fissure to the anterior end of the foramen of Monro. This approach consists of a more medial disconnection than was described by Delalande et al. Kawai et al. used an interhemispheric route through a microscope that does not provide optimum exposure to a lateral trajectory as compared to an endoscopic approach. The trajectory of our anterior disconnection is more peripheral than the one described by Kawai et al. Our anterior disconnection runs in a coronal plane starting at the junction of the genu and the $\mathrm{A}_{2}$ in the extraventricular plane and passes laterally to enter the ventricle. This plane of disconnection is outside the midline structures and ensures that no damage happens to them.

3. The middle disconnection is more or less the same as that described by Delalande et al. and Kawai et al. The middle disconnection runs in a parasagittal plane starting at the sphenoid ridge and passing posteriorly to the lateral part of the thalamus. It passes inferiorly such that it joins the temporal horn, thus converting the lateral ventricle and temporal horn into a single cavity.

4. Kawai et al. described cutting only fornix at the ventricular trigone. We have considered this transection in the posterior disconnection, which is a disconnection between the splenium and choroid plexus. This approach disconnects not just the fornix but also the tail of the hippocampus. This disconnection is performed until the arachnoid is exposed and ensures the disconnection of all of the so-called temporal efferents.

5. We have also described the excision of the ventral amygdala, which effectively disconnects the anterior part of the temporal lobe, which is tethered to the temporal stem through the dorsal amygdala. This technique was not described by Kawai et al. Delalande et al. suggested that the anterior part of the hippocampus should also be resected. This approach is not required because after the ventral amygdala is aspirated to the level of the 
beginning of the choroid plexus (plexal point), a complete disconnection is achieved.

6. As discussed by Kawai et al. and Delalande et al., the splenial disconnection should be checked carefully. We believe that a better disconnection of the splenium may be achieved if the procedure is performed with the use of the endoscope due to better visualization.

7. Whereas the underlying principles of disconnection are the same as those reported by Kawai et al. and Delalande et al., our technique has some modifications, which allow it to be better applied with the use of the endoscope and allow a greater degree of safety and efficacy.

8. In addition, the use of the endoscope reduces the amount of brain retraction and increases visualization and the safety of the surgery.

9. The location of the cranial opening was significantly smaller $(4 \times 3 \mathrm{~cm})$ and was located more anteriorly because the use of the endoscope allowed a greater depth of access.

We also introduced the nomenclature of the hybrid technique, which is in between the exoscopic system and endoscope-assisted surgery.

\section{Limitations of the Study}

The main limitation of this study is its observational nature, which does not allow class I evidence to objectively compare both techniques. However, by describing this technique created by the senior author, ${ }^{2,4}$ this study firmly establishes the role of endoscope-assisted hemispherotomy as a surgical technique demonstrating efficacy equal to $\mathrm{OH}$ while reducing blood loss and postoperative stay. It also paves the way for further use of endoscopic procedures to perform disconnective surgery for drug-resistant epilepsy.

\section{Conclusions}

Our study demonstrated the efficacy and safety of EH. In addition, $\mathrm{EH}$ has been shown to reduce blood loss and postoperative stay compared to $\mathrm{OH}$. The most common complication was fever, which was reactive in nature and subsided in most children within 1 week.

\section{Acknowledgments}

We acknowledge Mani Kalaiwani, MD, PhD, for statistical analysis. We are grateful to the patients and their families who were part of this study.

This study was supported by a grant (BT/01/COE/09/08) from the Department of Biotechnology, Government of India, the All India Institute of Medical Sciences of New Delhi and the National Brain Research Centre (the Centre of Excellence for Epilepsy and Magnetoencephalography), and the Indian Council of Medical Research (5/4-5/Neuro/2010-NCD-I).

\section{References}

1. Bahuleyan B, Manjila S, Robinson S, Cohen AR: Minimally invasive endoscopic transventricular hemispherotomy for medically intractable epilepsy: a new approach and cadaveric demonstration. J Neurosurg Pediatr 6:536-540, 2010

2. Baumgartner JE, Blount JP, Blauwblomme T, Chandra PS: Technical descriptions of four hemispherectomy approaches: from the Pediatric Epilepsy Surgery Meeting at Gothenburg 2014. Epilepsia 58 (Suppl 1):46-55, 2017

3. Carpay JA, Vermuelen J, Stroink H, Brouwer OF, Peters ACB, Aldenkamp AP, et al: Seizure severity in children with epilepsy: a parent-completed scale compared with clinical data. Epilepsia 38:346-352, 1997

4. Chandra PS, Kurwale N, Garg A, Dwivedi R, Malviya SV, Tripathi M: Endoscopy-assisted interhemispheric transcallosal hemispherotomy: preliminary description of a novel technique. Neurosurgery 76:485-495, 2015

5. Chandra PS, Padma VM, Shailesh G, Chandreshekar B, Sarkar C, Tripathi M: Hemispherotomy for intractable epilepsy. Neurol India 56:127-132, 2008

6. Chandra PS, Tripathi M: Endoscope-assisted hemispherotomy and corpus callostomy. J Neurosurg Pediatr 18:141, 2016 (Letter)

7. Chandra PS, Tripathi M: Epilepsy surgery: recommendations for India. Ann Indian Acad Neurol 13:87-93, 2010

8. Chandra SP, Tripathi M: Endoscopic epilepsy surgery: emergence of a new procedure. Neurol India 63:571-582, 2015

9. Chugani HT, Asano E, Juhász C, Kumar A, Kupsky WJ, Sood S: "Subtotal" hemispherectomy in children with intractable focal epilepsy. Epilepsia 55:1926-1933, 2014

10. Cook SW, Nguyen ST, Hu B, Yudovin S, Shields WD, Vinters $\mathrm{HV}$, et al: Cerebral hemispherectomy in pediatric patients with epilepsy: comparison of three techniques by pathological substrate in 115 patients. J Neurosurg 100 (2 Suppl Pediatrics):125-141, 2004

11. Daniel RT, Villemure JG: Hemispherotomy techniques. J Neurosurg 98:438-439, 2003

12. Delalande O, Bulteau C, Dellatolas G, Fohlen M, Jalin C, Buret V, et al: Vertical parasagittal hemispherotomy: surgical procedures and clinical long-term outcomes in a population of 83 children. Neurosurgery 60 (2 Suppl 1):ONS19ONS32, 2007

13. Delalande O, Dorfmüller G: [Parasagittal vertical hemispherotomy: surgical procedure.] Neurochirurgie 54:353357, 2008 (Fr)

14. Delalande O, Fohlen M, Bulteau C, Jalin C: Surgery for intractable focal epilepsy in children. Rev Neurol (Paris) 160:5S195-5S202, 2004

15. Delalande O, Pinard JM, Basdevant C, Gauthe M, Plouin P, Dulac O: Hemispherotomy: a new procedure for central disconnection. Epilepsia 33 (Suppl 3):99-100, 1992

16. Devlin AM, Cross JH, Harkness W, Chong WK, Harding B, Vargha-Khadem F, et al: Clinical outcomes of hemispherectomy for epilepsy in childhood and adolescence. Brain 126:556-566, 2003

17. Dorfer C, Czech T, Dressler A, Gröppel G, Mühlebner-Fahrngruber A, Novak K, et al: Vertical perithalamic hemispherotomy: a single-center experience in 40 pediatric patients with epilepsy. Epilepsia 54:1905-1912, 2013

18. Dwivedi R, Ramanujam B, Chandra PS, Sapra S, Gulati S, Kalaivani M, et al: Surgery for drug-resistant epilepsy in children. N Engl J Med 377:1639-1647, 2017

19. Frick AC, Falcone T: Robotics in gynecologic surgery. Minerva Ginecol 61:187-199, 2009

20. Granata T, Matricardi S, Ragona F, Freri E, Casazza M, Villani F, et al: Hemispherotomy in Rasmussen encephalitis: long-term outcome in an Italian series of 16 patients. Epilepsy Res 108:1106-1119, 2014

21. Griessenauer CJ, Salam S, Hendrix P, Patel DM, Tubbs RS, Blount JP, et al: Hemispherectomy for treatment of refractory epilepsy in the pediatric age group: a systematic review. J Neurosurg Pediatr 15:34-44, 2015

22. Hu WH, Zhang C, Zhang K, Shao XQ, Zhang JG: Hemispheric surgery for refractory epilepsy: a systematic review and meta-analysis with emphasis on seizure predictors and outcomes. J Neurosurg 124:952-961, 2016 
23. Jayakar P, Gaillard WD, Tripathi M, Libenson MH, Mathern GW, Cross JH: Diagnostic test utilization in evaluation for resective epilepsy surgery in children. Epilepsia 55:507-518, 2014

24. Kamath AA, Limbrick DL, Smyth MD: Characterization of postoperative fevers after hemispherotomy. Childs Nerv Syst 31:291-296, 2015

25. Kawai K, Morino M, Iwasaki M: Modification of vertical hemispherotomy for refractory epilepsy. Brain Dev 36:124129, 2014

26. Kovanda TJ, Rey-Dios R, Travnicek J, Cohen-Gadol AA: Modified periinsular hemispherotomy: operative anatomy and technical nuances. J Neurosurg Pediatr 13:332-338, 2014

27. Lew SM, Koop JI, Mueller WM, Matthews AE, Mallonee JC: Fifty consecutive hemispherectomies: outcomes, evolution of technique, complications, and lessons learned. Neurosurgery 74:182-195, 2014

28. Lindsay J, Ounsted C, Richards P: Hemispherectomy for childhood epilepsy: a 36-year study. Dev Med Child Neurol 29:592-600, 1987

29. Liu X, Otsuki T, Takahashi A, Kaido T: Vertical parasagittal hemispherotomy for Sturge-Weber syndrome in early infancy: case report and literature review. Springerplus 5:1434, 2016

30. Rasmussen T: Hemispherectomy for seizures revisited. Can J Neurol Sci 10:71-78, 1983

31. Rassweiler JJ, Autorino R, Klein J, Mottrie A, Goezen AS, Stolzenburg JU, et al: Future of robotic surgery in urology. BJU Int 120:822-841, 2017

32. Repperger DW: Adaptive displays and controllers using alternative feedback. Cyberpsychol Behav 7:645-652, 2004

33. Rydenhag B, Silander HC: Complications of epilepsy surgery after 654 procedures in Sweden, September 1990-1995: a multicenter study based on the Swedish National Epilepsy Surgery Register. Neurosurgery 49:51-57, 2001

34. Schramm J, Kuczaty S, Sassen R, Elger CE, von Lehe M: Pediatric functional hemispherectomy: outcome in 92 patients. Acta Neurochir (Wien) 154:2017-2028, 2012

35. Shimizu H, Maehara T: Modification of peri-insular hemispherotomy and surgical results. Neurosurgery 47:367-373, 2000

36. Sood S, Marupudi NI, Asano E, Haridas A, Ham SD: Endoscopic corpus callosotomy and hemispherotomy. J Neurosurg Pediatr 16:681-686, 2015

37. Tripathi M, Garg A, Gaikwad S, Bal CS, Chitra S, Prasad $\mathrm{K}$, et al: Intra-operative electrocorticography in lesional epilepsy. Epilepsy Res 89:133-141, 2010

38. Villemure JG, Daniel RT: Peri-insular hemispherotomy in paediatric epilepsy. Childs Nerv Syst 22:967-981, 2006

39. Villemure JG, Mascott CR: Peri-insular hemispherotomy: surgical principles and anatomy. Neurosurgery 37:975-981, 1995

40. Wiebe S, Berg AT: Big epilepsy surgery for little people: what's the full story on hemispherectomy? Neurology 80:232-233, 2013

41. Wieser HG, Blume WT, Fish D, Goldensohn E, Hufnagel A, King D, et al: ILAE Commission report. Proposal for a new classification of outcome with respect to epileptic seizures following epilepsy surgery. Epilepsia 42:282-286, 2001

42. Wilson PJE: Cerebral hemispherectomy for infantile hemiplegia. A report of 50 cases. Brain 93:147-180, 1970

\section{Disclosures}

The authors report no conflict of interest concerning the materials or methods used in this study or the findings specified in this paper.

\section{Author Contributions}

Conception and design: Chandra, Manjari Tripathi. Acquisition of data: Chandra, Subianto, Bajaj, Doddamani, Ramanujam, Garg, Madhavi Tripathi, Bal, Sarkar, Dwivedi, Sapra, Manjari Tripathi. Analysis and interpretation of data: Chandra, Subianto, Bajaj, Ramanujam, Chouhan, Garg, Madhavi Tripathi, Bal, Dwivedi, Manjari Tripathi. Drafting the article: Chandra, Subianto, Bajaj, Manjari Tripathi. Critically revising the article: Chandra, Bajaj, Girishan, Chouhan, Garg, Madhavi Tripathi, Sarkar, Sapra, Manjari Tripathi. Reviewed submitted version of manuscript: Chandra, Bajaj, Girishan, Doddamani, Ramanujam, Chouhan, Garg, Madhavi Tripathi, Bal, Sarkar, Dwivedi, Sapra, Manjari Tripathi. Approved the final version of the manuscript on behalf of all authors: Chandra. Statistical analysis: Subianto, Bajaj, Dwivedi. Administrative/technical/material support: Chandra, Chouhan, Garg, Madhavi Tripathi, Bal, Sarkar, Sapra, Manjari Tripathi. Study supervision: Chandra, Manjari Tripathi.

\section{Supplemental Information Videos}

Video 1. https://vimeo.com/287420691.

\section{Correspondence}

P. Sarat Chandra: All India Institute of Medical Sciences, New Delhi, India.saratpchandra3@gmail.com. 